\title{
Mini-symposium
}

\section{Myocardial hibernation and heart failure: introduction}

F or many years the functional sequelae of chronic coronary artery disease (CAD) were considered essentially irreversible and amenable only to palliative treatment. For example, asynergy on the left ventriculogram implied infarcted myocardium or scar. Evidence accrued over the past three decades, however, indicates that chronic left ventricular dysfunction in patients with CAD is not necessarily irreversible. ${ }^{1}$ Two pieces of evidence led to a new concept of reversible myocardial dysfunction with obvious and important implications for patient care. Firstly, clinicians and surgeons observed that chronic myocardial dysfunction present before coronary bypass often reversed following revascularisation. Secondly, studies by Gorlin and colleagues, ${ }^{2}$ using a catecholamine stress, showed that the asynergic left ventricle could improve its function with inotropic stimulation. This was the forerunner of dobutamine echocardiography, currently an important tool for detecting hibernating myocardium. ${ }^{1}$

Utilising these pieces of information in 1978 Diamond and colleagues ${ }^{3}$ presciently suggested the possibility that "ischemic non infarcted myocardium can exist in a state of function hibernation". Several years later Rahimtoola ${ }^{4}$ popularised the concept of hibernating myocardium and noted "there is a prolonged subacute or chronic stage of myocardial ischemia that is frequently not accompanied by pain and in which myocardial contractility and metabolism and ventricular function are reduced to match the reduced blood supply".

The debate on the pathophysiology of hibernating myocardium has attracted a lot of interest and, undoubtedly, has contributed significantly to stimulate new research on heart failure in patients with CAD. Although the debate is not over yet, some of the initial paradigms have been proven incorrect while new pathophysiological concepts have emerged. ${ }^{5}$

The concept of hibernating myocardium has resulted in a significant contribution to clinical cardiology because it pointed out that chronic left ventricular dysfunction can be reversible. Indeed, hibernating myocardium is frequently detected in patients with ischaemic heart failure, where its identification has provided a new rationale for coronary revascularisation.

This mini-symposium will deal with hibernation and heart failure, discuss some of the fundamental concepts in assessing myocardial viability, and finally highlight the role of surgical revascularisation in relation to hibernation.

P G Camici

MRC Clinical Sciences Centre, Hammersmith Hospital, London, UK paolo.camici@csc.mrc.ac.uk

\section{REFERENCES}

1 Wijns W, Vatner SF, Camici PG. Hibernating myocardium. N Engl J Med 1998;339:173-81.

2 Horn HR, Teichholz LE, Cohn PF, et al. Augmentation of left ventricular contraction pattern in coronary artery disease by an inotropic catecholamine. The epinephrine ventriculogram. Circulation 1974:49:1063-71.

3 Diamond GA, Forrester JS, deLuz PL, et al Postextrasystolic potentiation of ischemic myocardium by atrial stimulation. Am Heart J 1978:95:204-9.

4 Rahimtoola SH. A perspective on the three large multicenter randomized clinical trials of coronary bypass surgery for chronic stable angina. Circulation 1985.72.V123-35.

5 Camici PG, Rimoldi OE. Myocardial blood flow in patients with hibernating myocardium. Cardiovasc Res 2003:57:302-11.

\section{IMAGES IN CARDIOLOGY}

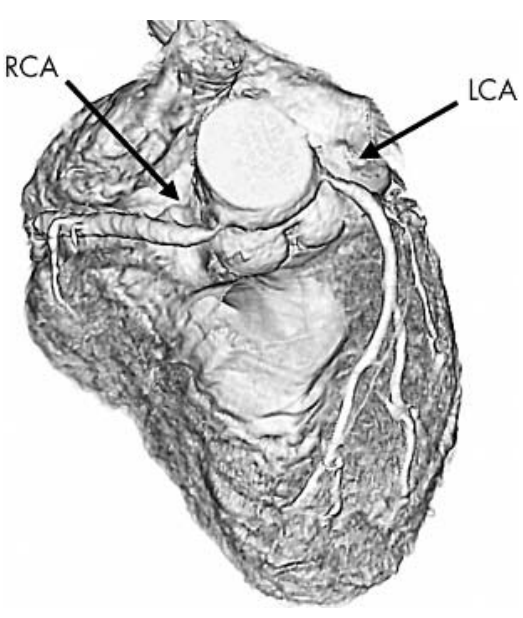

A 34 year old man presented with a five month history of exertional chest discomfort on moderate exercise. He had no significant coronary risk factors. A diagnosis of Takayasu's arteritis had been made three years before when he had undergone aortic arch replacement for his thoracic aortic aneurysm. Multislice computed tomography (left anterior oblique view depicting severe ostial stenosis of both right (RCA) and left (LCA) coronary arteries) revealed severe ostial disease of both right and left coronary arteries. A conventional aortocoronary angiogram was compatible with this finding. Elective coronary bypass grafting was subsequently performed successfully.

On the basis of postmortem examinations the incidence of coronary arterial lesions in Takayasu's arteritis has been reported at approximately $10 \%$. Lesions are most commonly confined to the ostial and proximal portions of the coronary arteries. In cases having this type of coronary involvement, an imprudent selective coronary angiography or maximum exercise test should be avoided. When chest symptoms are observed in this disorder, this non-invasive imaging modality may be the first choice before invasive and risky tests. 